\title{
Effect of carrier gas on GaN epilayer characteristics
}

\author{
Y. S. Cho ${ }^{*}$, H. Hardtdegen, N. Kaluza, N. Thillosen, R. Steins, Z. Sofer, and H. Lüth \\ Institute of Thin Films and Interfaces (ISG-1), Center of Nanoelectronic Systems for Information \\ Technology, Research Center Jülich, 52425 Jülich, Germany
}

Received 25 July 2005, revised 5 January 2006, accepted 16 January 2006

Published online 10 May 2006

PACS 61.10.Nz, 68.55.Jk, 68.37.Ps, 78.55.Cr, 81.05.Ea, 81.15.Kk

Metalorganic vapor phase epitaxy (MOVPE) of GaN was performed using hydrogen $\left(\mathrm{H}_{2}\right)$, nitrogen $\left(\mathrm{N}_{2}\right)$ and $\mathrm{H}_{2} / \mathrm{N}_{2}$ mixtures thereof as the carrier gas in the high temperature buffer growth range. The effect of carrier gas on the structural and morphological characteristics of the epilayers was systematically studied using interference and atomic force microscopy (AFM), photoluminescence (PL) measurements at $2 \mathrm{~K}$, Raman spectroscopy and X-ray diffraction (XRD). The higher the $\mathrm{N}_{2}$ content in the carrier gas, the more pinholes are observed, the lower compressive strain and the higher dislocation density in the layers. A carrier gas composition range was defined at which $\mathrm{GaN}$ layers with acceptable structural and morphological characteristics are achieved.

(C) 2006 WILEY-VCH Verlag GmbH \& Co. KGaA, Weinheim

\section{Introduction}

Despite their high dislocation density, GaN and related nitride compounds are extremely important materials for device applications in light emitting diodes (LEDs) [1], laser diodes (LDs) [2] and high power and high frequency devices such as high electron mobility transistors (HEMTs) [3]. In order to grow high quality GaN epilayers, a number of growth parameters have to be optimized and their influence on growth need to be studied especially with respect to their influence on structural characteristics. Even though the effect of most of the growth parameters on epilayer characteristics has been clarified the role of the carrier gas is still discussed quite contradictorily. For the growth of $\mathrm{GaN}$, most groups reported the use of hydrogen $\left(\mathrm{H}_{2}\right)$ as carrier gas. Amano et al. used nitrogen $\left(\mathrm{N}_{2}\right)$ as the ambient gas [4]. Nakamura et al. reported the use of a gas mixture of $\mathrm{H}_{2}$ and $\mathrm{N}_{2}$ as the sub-flow gas [5]. These GaN epilayers grown in the presence of $\mathrm{N}_{2}$ show good uniformity and high quality. However, some other groups reported that pure $\mathrm{N}_{2}$ is not only detrimental to the morphology and the structural quality of $\mathrm{GaN}$ but also to the electrical properties [6]. Details of the underlying physical processes, however, are not yet understood.

In this paper, the influences of $\mathrm{H}_{2}$ and $\mathrm{N}_{2}$ as well as $\mathrm{H}_{2} / \mathrm{N}_{2}$ carrier gas mixtures are discussed on the morphological and structural characteristics of GaN epilayers. The study is carried out to clarify why metalorganic vapor phase epitaxy (MOVPE) of $\mathrm{GaN}$ in $\mathrm{N}_{2}$ atmosphere differs from that in $\mathrm{H}_{2}$.

\section{Experiment}

The growth of undoped GaN epilayers was performed on sapphire (0001) substrates by MOVPE in an AIX 200/4 RF-S horizontal reactor. The samples were grown using the inverted inlet process [7] using trimethylgallium (TMGa) and ammonia $\left(\mathrm{NH}_{3}\right)$ as the $\mathrm{Ga}$ and $\mathrm{N}$ precursors, respectively. The growth pressure was maintained at 200 mbar. For all samples a $25 \mathrm{~nm}$ thick low temperature GaN nucleation layer (LT-GaN NL) was deposited at $550{ }^{\circ} \mathrm{C}$ in $\mathrm{H}_{2}$ environment. The LT-GaN NL was annealed at

\footnotetext{
* Corresponding author: e-mail: y.s.cho@fz-juelich.de, Phone: +49 246161 2991, Fax: +49 2461612940
} 
$970{ }^{\circ} \mathrm{C}$ for 10 minutes also in $\mathrm{H}_{2}$ carrier gas and ammonia. After the annealing process, growth was resumed and the successive high temperature growth step was performed with different ratios of $\mathrm{H}_{2} / \mathrm{N}_{2}$ carrier gas mixtures - $0 \%, 50 \%, 70 \%, 80 \%$ and $100 \%$ - at $1100{ }^{\circ} \mathrm{C}$ for 60 minutes while the total flow was kept constant at $9 \mathrm{slm}$. Since the carrier gas had a strong influence on the true surface temperature [8] - the surface temperature drops successively by $50{ }^{\circ} \mathrm{C}$ from the pure $\mathrm{H}_{2}$ carrier gas to the pure $\mathrm{N}_{2}$ carrier gas sample -, heating control temperature was adjusted in order to compensate for this effect and to achieve the same surface temperature $1100{ }^{\circ} \mathrm{C}$ for all five samples.

Growth was monitored by in-situ reflectometry combined with emissivity corrected pyrometry (EpiRDA (TT)). The surface morphology was observed ex-situ by Nomarski- on the micrometer scale and atomic force microscopy (AFM) on the nanometer scale. The structural characteristics of the layers with respect to strain were studied at first by PL at $2 \mathrm{~K}$ using a $\mathrm{He}-\mathrm{Cd}$ laser with $325 \mathrm{~nm}$ wavelength and by identifying the excitonic line position. The penetration depth of the laser is estimated to be approximately $100 \mathrm{~nm}$. Since the substrates are transparent, the measurements were carried out from the backside of the wafer and from the front-side giving access to the strain state of the first $100 \mathrm{~nm}$ and the last $100 \mathrm{~nm}$ of GaN growth, respectively. The in-plane biaxial stress of the GaN epilayers was analyzed by Raman spectroscopy at room temperature. At last the structural characteristics of the $\mathrm{GaN}$ epilayers were evaluated by X-ray diffraction (XRD) using the symmetric (002) and (110) and asymmetric (102) reflection the full width at half maximum (FWHM) of which are sensitive to screw and mixed types of threading dislocations (TDs), to pure edge and mixed types of TDs, and to all types of TDs, respectively [9].

\section{Results and discussion}

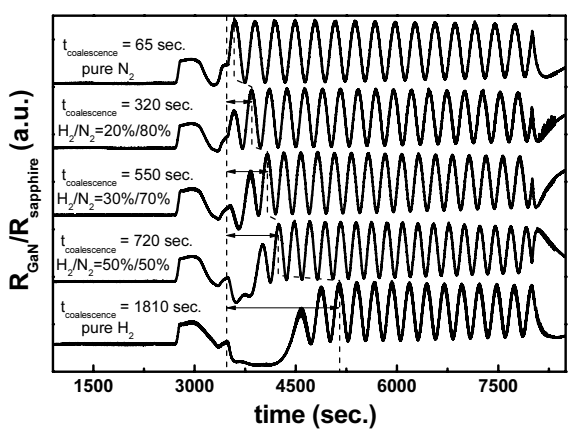

Fig. 1 The effect of $\mathrm{N}_{2}$ on the coalescence time and thickness. With increasing $\mathrm{N}_{2}$ content in the carrier gas, the coalescence time (and therefore the "coalescence thickness") decreases drastically.

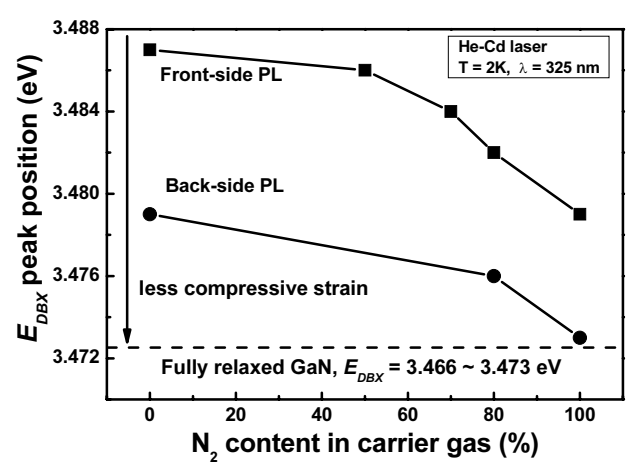

Fig. 2 The comparison of front- and back-side PL measurement. At the interface between GaN epilayer and sapphire substrate, GaN has less compressive strain than the surface of $\mathrm{GaN}$ epilayer.

Figure 1 shows the reflectance transients at $600 \mathrm{~nm}$ taken during growth for the sample series. Three different growth regimes are observed. Before the first dotted line the identical growth steps for all samples are to be seen: the LT-GaN NL growth and the annealing phase. After the first dotted line, the coalescence phase starts in which the nuclei grow together. Gradually oscillations form which reach a constant intensity. The second dotted line denotes the end of the coalescence phase and the onset of complete $2 \mathrm{D}$ growth. With increasing $\mathrm{N}_{2}$ content in the gas phase (from bottom to top) the coalescence time and therefore the "coalescence thickness" decreases drastically from 1810 to 65 seconds. Also the growth rate varies and therefore the layer thickness changes from 2.29 to $1.73 \mu \mathrm{m}$. Earlier reports point out that the coalescence phase strongly influences the strain and the number of threading dislocations as well as the point defects in the layer as well as the surface morphology [10]. Therefore the morphology of the layers was investigated next.

Although somewhat contradictorily to observations made by other groups [6], the morphology on the micrometer scale is smooth and specular. On the nanometer scale, however, the surface morphology is 
quite different. For pure $\mathrm{H}_{2}$ carrier gas, the surface exhibits the well known straight atomic steps. However, the higher the $\mathrm{N}_{2}$ content the more the steps become round and pits are observed. This may be due to a different surface stoichiometry in $\mathrm{H}_{2}$ and $\mathrm{N}_{2}$ carrier gas as has been observed surface photoabsorption (SPA) spectra [11]. However still studies need to be carried out which relate the spectra to surface reconstructions.

Since the morphology development in the coalescence phase is closely related to the compressive strain in the layer [12], the strain was investigated next. The GaN band gap increases in case of biaxial in-plane compression compared to fully relaxed $\mathrm{GaN}$. The donor bound exciton energy $\left(E_{D B X}\right)$ therefore increases with increasing compressive strain in the layers. In Fig. 2 the $E_{D B X}$ is plotted as a function of nitrogen content for PL performed on the back-side and on the front-side of the sample. The value for fully relaxed $\mathrm{GaN}$ according to reference values $[13,14]$ is also included. The results of back-side PL reveal that near the interface between GaN epilayer and sapphire substrate there is less compressive strain. There are more point defects near the interface due to LT-GaN nucleation layer, therefore it has less compressive strain than at the surface. For both front- and backside measurements the position of the $E_{D B X}$ gradually moves towards lower energies with increasing nitrogen content as the layers show decreasing compressive strain. However above $70 \% \mathrm{~N}_{2}$ content the observed strain relaxation becomes bigger. The decrease in coalescence thickness with increasing nitrogen content leads to early strain relaxation. The coalescence time correlates with the size of the nuclei before neighboring grains coalesce: the smaller the thickness the smaller the grain size and the more dislocations can be found. Also it becomes apparent that the strain is smaller close to the substrate compared to near the surface. It means that thermally induced stress for non-coalesced films should be very small [12].

Raman measurements are well suited for the non-destructive characterization of the overall stress in the materials. The intense $E_{2}$ phonon mode of $\mathrm{GaN}$ was chosen as a reference peak for the strain determination. Figure 3 shows the Raman shift of the $E_{2}$ phonon mode and biaxial stress in the layers as a function of nitrogen content in the carrier gas. It was reported by Koh et al. [15] that in-plane biaxial stress $(\sigma)$ is given by

$$
\sigma[G P a]=-0.22 \times\left(\omega-\omega_{0}\right),
$$

where $\omega$ is the measured $E_{2}$ phonon mode for a sample and $\omega_{0}$ is that for the reference sample, $\omega_{0}\left(E_{2}\right)=$ $566.34 \mathrm{~cm}^{-1}$. Our reference sample was a $300-\mu \mathrm{m}$-thick free-standing $\mathrm{GaN}$ substrate prepared by hydride vapor phase epitaxy (HVPE). The commercial reference sample exhibited a TD density less then $1 \times 10^{8}$ $\mathrm{cm}^{-2}$ and resistivity less then $30 \mathrm{M} \Omega \cdot \mathrm{cm}$. At first the Raman shift and biaxial stress gradually decrease with increasing nitrogen content. Above $70 \%$ nitrogen content the decrease becomes more sudden. It should be noted in Fig. 3 that the Raman shift and the biaxial stress are minimal for the GaN epilayer grown in pure $\mathrm{N}_{2}$ carrier gas. These Raman results agree well with PL results. Also these Raman and PL results are well matched with previous reports [16]. It means that we can influence the stress using carrier gas. The structural quality was investigated with XRD at last.

The FWHM of XRD $\omega$-scans for the (002), (110) and (102) reflections are presented in Fig. 4. The FWHM of all three reflections increase with increasing $\mathrm{N}_{2}$ content in carrier gas indicating an increase of TDs. Above $70 \% \mathrm{~N}_{2}$ content in carrier gas, the increase becomes stronger for the (110) and (102) reflections compared to the (002) reflection. Obviously, mainly the edge dislocation density increases with increasing $\mathrm{N}_{2}$ content. The investigations show that a minimal amount of $\mathrm{H}_{2}$ is necessary in the reactor in order to obtain layers of sufficient structural and morphological quality. Apparently the higher edge dislocation density correlates with strain relaxation due to the coalescence of small grains in the early stage of high temperature growth [10]. By intentionally using carrier gas mixtures the strain in the layers can be tailored. In future transmission electron microscopy (TEM) must still be carried out to confirm the results observed.

\section{Conclusions}

MOVPE of GaN was performed using $\mathrm{H}_{2}, \mathrm{~N}_{2}$ and $\mathrm{H}_{2} / \mathrm{N}_{2}$ mixtures thereof as the carrier gas in the high temperature buffer growth range. Based on the overall results, an increase of $\mathrm{N}_{2}$ content in the carrier gas 
leads to a change in the surface morphology of the GaN epilayers on the nanometer scale. The shortened coalescence time affects the TD density in a detrimental way to the layers and reduces the compressive strain. A sufficient structural and morphological quality of the layers can nevertheless be obtained as long as the $\mathrm{H}_{2}$ content in the gas phase is at least $30 \%$ in the coalescence phase of growth. The influence of carrier gas on strain can be employed to tailor the strain in the layers which is an important issue in fabrication of optical and electrical devices. Further TEM studies are necessary to study the evolution of TDs and point defects during growth.

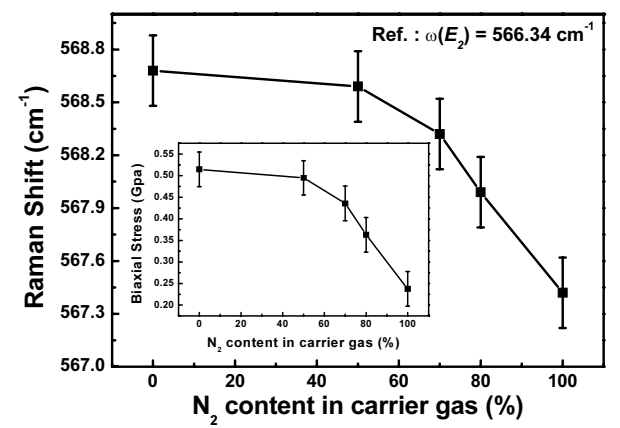

Fig. 3 Raman shift of the $E_{2}$ phonon mode and biaxial stress in the $\mathrm{GaN}$ epilayer.

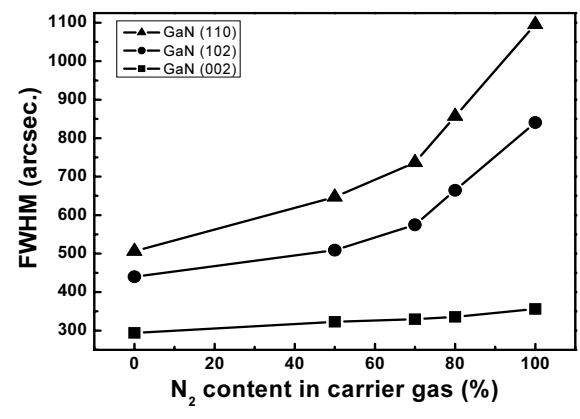

Fig. 4 FWHM of X-ray rocking curves of the GaN epilayers.

Acknowledgements The authors would like to thank Dr. M. von der Ahe and Dr. E. Hollmann for fruitful discussion about XRD measurement, Dr. G. Crecelius for assistance with Raman and K. Wirtz for the technical support as well as German Academic Exchange Service (DAAD) for the research grant for Ph. D. degree to Y. S. Cho.

\section{References}

[1] T. Mukai, H. Narimatsu, and S. Nakamura, Jpn. J. Appl. Phys. 37, L479 (1998).

[2] S. Nakamura, M. Senoh, S. Nagahama, T. Matsushita, H. Kiyoku, Y. Sugimoto, T. Kozaki, H. Umemoto, M. Sano, and T. Mukai, Jpn. J. Appl. Phys. 38, L226 (1999).

[3] P. Javorka, A. Alam, M. Wolter, A. Fox, M. Marso, M. Heuken, H. Lüth, and P. Kordoš, IEEE Electron Device Lett. 23, 287 (2002).

[4] H. Amano, N. Sawaki, and I. Akasaki, J. Cryst. Growth 68, 163 (1984).

[5] S. Nakamura, Y. Harada, and M. Senoh, Appl. Phys. Lett. 58, 2021 (1991).

[6] H. X. Wang, Y. Amijima, Y. Ishihama, and S. Sakai, J. Cryst. Growth 233, 681 (2001).

[7] H. Hardtdegen, N. Kaluza, R. Steins, R. Schmidt, K. Wirtz, E. V. Yakovlev, R. A. Talalaev, and Yu. N. Makarov, J. Cryst. Growth 272, 407 (2004).

[8] H. Hardtdegen, N. Kaluza, R. Steins, Y. S. Cho, Z. Sofer, M. Zorn, K. Haberland, and J.-T. Zettler, phys. stat. sol. (b) 242, 2581 (2005).

[9] B. Heying, X. H. Wu, S. Keller, Y. Li, D. Kapolnek, B. P. Keller, S. P. DenBaars, and J. S. Speck, Appl. Phys. Lett. 68, 643 (1996).

[10] T. Böttcher, E. Einfeldt, S. Figge, R. Chierchia, H. Heinke, D. Hommel, and J. S. Speck, Appl. Phys. Lett. 78, 1976 (2001).

[11] Y. Kobayashi and N. Kobayashi, J. Cryst. Growth 189/190, 301 (1998).

[12] S. Einfeldt, T. Böttcher, S. Figge, and D. Hommel, J. Cryst. Growth 230, 357 (2001).

[13] B. Monemar, Phys. Rev. B 10, 676 (1974).

[14] K. Kornitzer, T. Ebner, K. Thonke, R. Sauer, C. Kirchner, M. Kamp, V. Schwegler, M. Leszczynski, I. Grzegory, and S. Porowski, Phys. Rev. B 60, 1471 (1999).

[15] E. K. Koh, Y. J. Park, E. K. Kim, C. S. Park, S. H. Lee, J. H. Lee, and S. H. Choh, J. Cryst. Growth 218, 214 (2000).

[16] D. Li, H. Chen, H. B. Yu, Y.J. Han, X. H. Zheng, Q. Huang, and J. M. Zhou, J. Cryst. Growth 263, 76 (2004). 